\title{
REDES DE ATENÇÃO A SAÚDE: A EXPERIÊNCIA DO TELESSAÚDE UERJ
}

\author{
Networks for Health Care: UERJ Telehealth Center Results
}

\begin{abstract}
Alexandra Maria Vieira Monteiro; ; João Paulo Pires das Neves²; Edson Paulo Diniz³; Marta do Nascimento Rocha4; Rafael Pablo Sanzana Batista5; Rodrigo de Souza Santos ${ }^{6}$; Bruno Silveira Krause7; Frederico Sá da Silva $;$ Munique Valério Bara dos Santos ${ }^{9}$; William dos Santos Ribeiro Junior ${ }^{10}$
\end{abstract}

\begin{abstract}
Resumo Objetivos: Apresentar os resultados do Telessaúde UERJ nas Redes de Atenção a Saúde (RAS). Materiais e métodos: Análise transversal qualitativa e quantitativa. Resultados: 27.766 profissionais, $99.4 \%$ brasileiros, $67 \%$ do sudeste e $36.09 \%$ enfermeiros. A RAS Primária foi prevalente $(89,3 \%)$ sobre as demais. Conclusão: A telessaúde apóia a organização das RAS.

Palavras-chave: Telemedicina, Telessaúde, Atenção a Saúde
\end{abstract}

Abstract Aims: To present the results of UERJ Telehealth Center Networks in Networks for Health Care. Materials and methods: qualitative and quantitative cross-sectional analysis. Results: 27,766 professionals, $99.4 \%$ of them Brazilian, $67 \%$ from Southeast of Brazil and $36.09 \%$, nurses. The RAS Primary was prevalent (88.7\%) on the others. Conclusion: Telehealth supports the organization of RAS. Keywords: Telemedicine, Telehealth, Attention to Health

\section{Introdução e Objetivos}

A criação da Internet gerou um grau inaudito de conexão irrestrita entre as pessoas tornando inexorável a incorporação do uso das tecnologias de informação e de comunicação, também, na saúde. Com esta visão, o Laboratório de Telessaúde ${ }^{1}$, da Universidade do Estado do Rio de Janeiro (UERJ), vem atuando em diferentes projetos ${ }^{2-11}$, desde a sua criação. Dentre estes, destaca-se o Núcleo Rio de Janeiro ${ }^{12}$ - Programa Nacional Telessaúde Brasil Redes ${ }^{13}$ e o Núcleo UERJ - Rede Universitária de Telemedicina ${ }^{14}$ pelas suas ações nas redes de atenção a saúde. Redes de Atenção à Saúde (RAS) $)^{15,16}$ são definidas como uma estratégia para um cuidado integral e direcionado as necessidades de saúde da população. Estas, constituem-se em arranjos organizativos formados por ações e serviços de saúde com diferentes configurações tecnológicas e missões assistenciais. O objetivo deste artigo é apresentar os resultados qualitativos e quantitativos das ações do Telessaúde UERJ nas Redes de Atenção a Saúde.

\section{Materiais e Métodos}

As atividades do Telessaúde UERJ podem ser agrupadas, de forma sucinta, em síncronas e assíncronas. Para as atividades síncronas são utilizados tecnologias

\footnotetext{
1. Professora Associado da Faculdade de Ciências Médicas; Coordenadora do Laboratório de Telessaúde - Universidade do Estado do Rio de Janeiro. - Doutor em Medicina; 2. Gerente de Criação e Desenvolvimento do Telessaúde UERJ; 3. Gerente de Informática do Telessaúde UERJ; 4. Relações Públicas do Telessaúde UERJ; 5. Desenvolvedor Web do Telessaúde UERJ; 6. Desenvolvedor Web do Telessaúde UERJ; 7. Suporte Técnico do Telessaúde UERJ; 8. Suporte Técnico do Telessaúde UERJ; 9 . Suporte Técnico do Telessaúde UERJ; 10. Suporte Técnico do Telessaúde UERJ. Endereço Eletrônico: coordenacao@telessaude.uerj.br (Alexandra Maria Vieira Monteiro).
} 
para teleconferência em baixa velocidade (webconferências), em alta velocidade (videoconferência) e em alta definição (telepresença) e um serviço de chat como facilitador para suporte em informática e para a teleconsultoria em saúde. Todas as atividades de tele-educação síncronas, incluindo aulas e seminários multiprofissionais temáticos, são gravadas e disponibilizadas para a reutilização dos usuários. Estão disponíveis, também, cursos a distância para profissionais de saúde de nível médio e superior. Para o acesso dos profissionais de saúde é necessário um cadastro único em um ambiente virtual (Moodle; Martins Dougiamas, Perth, Austrália) que foi customizada pela equipe do Laboratório de Telessaúde da UERJ (Figura 1).

O sistema de apoio a teleconsultoria em saúde, desenvolvido pela equipe do Telessaúde UERJ está contido dentro do ambiente virtual Moodle customizado e pode ser acessado para teleconsultoria síncrona e assíncrona, conforme ilustra o infográfico abaixo (Figura 2).

\section{Resultados}

Até o presente momento, 27.766 profissionais de saúde estão cadastrados na plataforma do Telessaúde UERJ. Destes, $99.4 \%$ são brasileiros e $0.6 \%$ estrangeiros localizados em diferentes continentes (Figura 3).
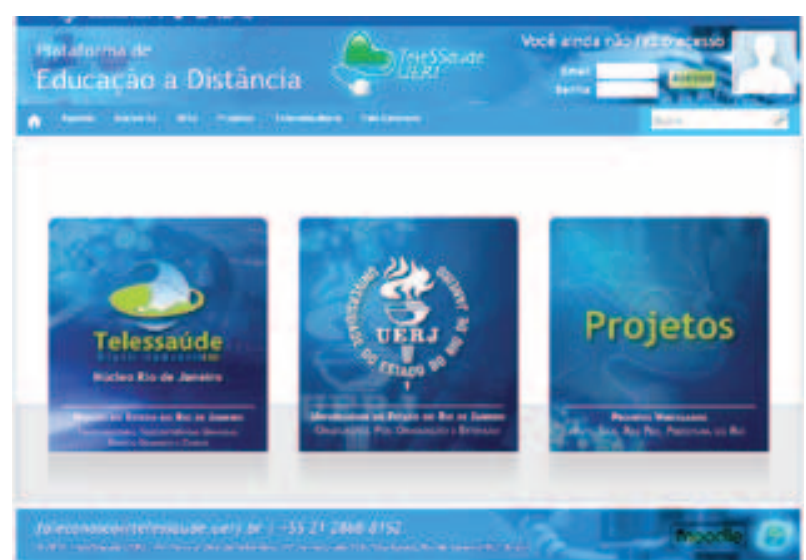

\section{Figura 1:}

Ambiente virtual do Telessaúde Uerj

[Acesso disponível em http://www.telessaude.uerj.br/ava/]

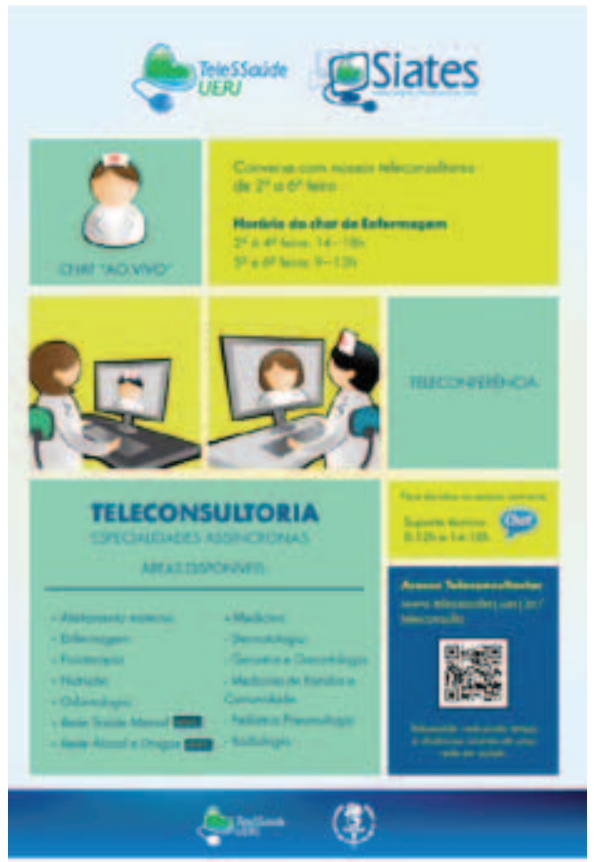

Figura 2: SIATES Sistema Apoio de Teleconsultoria em Saúde (em setembro de 2012).

Figura 3: Distribuição geográfica dos usuários cadastrados no Brasil e em outros países. 
No grupo de brasileiros a abrangência é nacional, com prevalência de usuários na região sudeste (67\%), seguido pela região nordeste (20\%), sul (6\%), centro-oeste (5\%) e norte (2\%). Dentre as profissões, os enfermeiros são prevalentes (36.09\%), seguidos por fisioterapeutas (21.56\%), nutricionistas (8.75\%), médicos (5.91\%) e cirurgiões-dentistas (2.44\%); dentre as outras profissões em saúde.

Dentre as atividades de tele-educação, os seminários multidisciplinares temáticos síncronos são os mais acessados (67.8\%), seguidos pelos cursos à distância (29.9\%), dentre as outras atividades. Em relação ao total de teleconsultorias houve predomínio da síncrona (75.4\%) sobre a assíncrona (24.6\%) e para o telediagnóstico em radiologia foi utilizado somente o sistema assíncrono.

Nas Redes de Atenção a Saúde há prevalência de participação na Rede Atenção Primária (88,7\%), seguida pela Rede Urgência e Emergência (3,7\%), Rede Cegonha (3.2\%), Saúde Bucal (2.3\%) e Rede Saúde Mental e Álcool e Drogas $(2,1 \%)$, que foram iniciadas recentemente.

\section{Discussão}

Embora sejam inegáveis e representativos os avanços alcançados na saúde pelos governos municipal, estadual e federal nos últimos anos, torna-se cada vez mais evidente a dificuldade em superar as barreiras geográficas, a segmentação das ações e serviços de saúde e qualificar, de forma continuada, o atendimento profissional no contexto atual. Nesta perspectiva, as Redes de Atenção à Saúde (RAS) têm por objetivo atuar como estratégia para superar a fragmentação da atenção e da gestão nas Regiões de Saúde e aperfeiçoar o funcionamento político-institucional do Sistema Único de Saúde (SUS) com vistas a assegurar ao usuário o conjunto de ações e serviços que necessita com efetividade e eficiência. Experiências têm demonstrado que a organização da RAS tendo a atenção primária como base do cuidado da rede, se apresenta como um mecanismo de superação da fragmentação sistêmica; são mais eficazes, tanto em termos de organização interna quanto em sua capacidade de fazer face aos atuais desafios do cenário socioeconômico, demográfico, epidemiológico e sanitário ${ }^{15,16}$. Os resultados apresentados neste artigo demonstram uma demanda espontânea, pelos profissionais, pela Rede de Atenção Primária. Este comportamento é verificado tanto nas atividades de tele-educação como nas de teleassistência (teleconsultoria). Nesta linha, a experiência exitosa global do Programa Nacional Telessaúde Brasil Redes ${ }^{17}$ e a do próprio Telessaúde UERJ ${ }^{18}$, anteriormente assinalada, destacam a relevância e o impacto da utilização das tecnologias de informação e de comunicação como apoio às RAS, notadamente a Rede de Atenção Primária, bem como apontam resultados positivos na eliminação das barreiras geográficas, na educação e atualização permanente dos profissionais e como apoio aos processos das e serviços em saúde. Por outro lado, a integração com os grupos de trabalho atuantes na Rede RUTE ${ }^{14}$, aproximando a academia do serviço, conectando municípios geograficamente afastados a centros de excelência internacionais e facilitando a troca de experiências está, irrefutavelmente, contribuindo para a qualificação profissional considerando critérios de acesso, escala e escopo.

\section{Conclusão}

A experiência da incorporação da telessaúde, definitivamente, está modificando paradigmas para a democratização da saúde na perspectiva da universalização, da efetividade e da qualificação das ações.

\section{Referências}

1. Portal do Laboratório de Telessaúde da Uerj [Internet]. Rio de Janeiro: Universidade do Estado do Rio de Janeiro, Hospital Universitário Pedro Ernesto; 2011 [atualizado em 2012; acesso em 17/09/2012]. Disponível em http://www.telessaude.uerj.br/site/.

2. Monteiro AMV, Corrêa DG, Santos AAS, Cavalcanti SA, Sakuno T, Filgueiras T, et al. Telemedicine and Pediatric Radiology: A New Environment for Training, Learning, and Interactive Discussions. TelemedJEHealth. 2011 Dec.;17(10):753-6. 
3. Grande J, Bhaya A, Monteiro AMV, Ferreira LV, Bahia P, Santos AAS. Digitalização de Filmes Radiográficos com Costura de Imagens. Radiol Bras. 2011 Jul;44(4): 233-7.

4. Monteiro AM, Corrêa DG . The Brazilian Pediatric Radiology Telemedicine Program: an education tool connecting people around the world. DI Europe.2012 Apr-May; 48-9.

5. Santos M, Santos R, Neves JPN, Rocha M, Diniz E, Monteiro AM. Telehealth UERJ RJ-Brazil: Innovating by the fusion of technologies for teleconferences. In: Malina Jordanova and Frank Lievens. Global Telemedicine and eHealth Updates Knowledge Resources. Vol. 5. Luxembourg: G.D. of Luxembourg; 2012.p. 148-150.

6. Rocha M, Neves JPN, Santos R, Santos M, Diniz E, Monteiro AM. Social Networks and Telehealth: Telehealth UERJ-Brazil s experience in Preventing Childhood Obesity at Schools. In: Malina Jordanova and Frank Lievens. Global Telemedicine and eHealth Updates Knowledge Resources. Vol. 5. Luxembourg: G.D. of Luxembourg; 2012. p. 281-283.

7. Monteiro AM, Diniz E, Santos R, Neves JPN, Santos M, Neves AL. Teleradiology at Emergency Units in Rio de Janeiro: reporting Telehealth UERJ-Brazil experience on the issue. In: Malina Jordanova and Frank Lievens. Global Telemedicine and eHealth Updates Knowledge Resources. Vol. 5.Luxembourg: G.D. of Luxembourg; 2012. p. 297-299.

8. Santos M, Santos R, Neves JPN, Rocha M, Diniz E, Monteiro AM. Telehealth as professional update: the experience of Telehealth UERJ Brazil Networks Program in Distance Education Course. In: Malina Jordanova and Frank Lievens. Global Telemedicine and eHealth Updates Knowledge Resources. Vol. 5. Luxembourg: G.D. of Luxembourg, 2012. p. 334-337.

9. Santos R, Santos M, Neves JPN, Rocha M, Diniz E, Monteiro AM. Telehealth UERJ RJ-Brazil Networks: the impact of using technologies for teleconsulting. In: Malina Jordanova and Frank Lievens. Global Telemedicine and eHealth Updates Knowledge Resources. Vol. 5. Luxembourg: G.D. of Luxembourg; 2012. p. 338-340.

10. Diniz E, Rocha M, Santos R, Neves JPN, Santos M, Monteiro AM. Telehealth UERJ RJ-Brazil: the impact of using technologies for health education. In: Malina Jordanova and Frank Lievens. Global Telemedicine and eHealth Updates Knowledge Resources. Vol. 5. Luxembourg: G.D. of Luxembourg; 2012. p. 341-344.
11. Diniz E, Santos M, Santos R, Rocha M, Neves JPN, Monteiro AM. University Network in Telemedicine (RUTE) and Telehealth Brazililian Networks: Telehealth UERJ Center RJBrazil. In: Malina Jordanova and Frank Lievens. Global Telemedicine and eHealth Updates Knowledge Resources. Vol. 5. Luxembourg: G.D. of Luxembourg; 2012. p. 351-354.

12. Núcleo Rio de Janeiro - Programa Nacional Telessaúde Brasil Redes [Internet]. Rio de Janeiro: Universidade do Estado do Rio de Janeiro, Hospital Universitário Pedro Ernesto; 2011 [atualizado em 2012; acesso em 17/09/2012]. Disponível em http://www.telessauderj.uerj.br/site/.

13. Brasil. Ministério da Saúde. PORTARIA № 2.546, DE 27 DE OUTUBRO DE 2011. Redefine e amplia o Programa Telessaúde Brasil, que passa a ser denominado Programa Nacional Telessaúde Brasil Redes (Telessaúde Brasil Redes). Diário Oficial da União. 2011 Out 28; Seção 1. Brasília; 2011.

14. Rede Universitária de Telemedicina (RUTE) - Rede Nacional de Ensino e Pesquisa (RNP) [Internet]. Rio de Janeiro: Universidade do Estado do Rio de Janeiro, Hospital Univeristário Pedro Ernesto; 2011 [acesso em: 17/09/2012].

15. Brasil. Ministério da Saúde. PORTARIA № 4.279, DE 30 DE DEZEMBRO DE 2010. Estabelece as diretrizes para a organização da Rede de Atenção à Saúde no Sistema Único de Saúde (SUS). Diário Oficial da União. 2010 Dez 31; Seção 1. Brasília; 2010.

16. Brasil. Ministério da Saúde. PORTARIA № 2.546, DE 27 DE OUTUBRO DE 2011. Redefine e amplia o Programa Telessaúde Brasil, que passa a ser denominado Programa Nacional Telessaúde Brasil Redes (Telessaúde Brasil Redes). Diário Oficial da União. 2011 Out 28; Seção 1. Brasília; 2011.

17. Haddad AE. Experiência Brasileira do Programa Nacional Telessaude Brasil. In Mathias I and Monteiro A. Gold Book [on-line]: inovação tecnológica em educação e saúde. Rio de Janeiro: EdUERJ; 2012 Ago [Acesso em 26/09/2012]. Disponível em: http://www.telessaude.uerj.br/goldbook/artigos/.

18. Monteiro AMV, Krause B, Diniz EP, Silva FS, Neves JPP, Rocha M, Santos MS, Santos R, Santos W. Telessaúde UERJ. In Mathias I and Monteiro A. Gold Book [on-line]: inovação tecnológica em educação e saúde. Rio de Janeiro: EdUERJ; 2012 Ago [Acesso em 26/09/2012]. Disponível em: http://www.telessaude.uerj.br/goldbook/artigos/. 